Acta Univ. Sapientiae, Mathematica, 13, 1 (2021) 127-144

DOI: $10.2478 /$ ausm-2021-0007

\title{
On a new one-parameter generalization of dual-complex Jacobsthal numbers
}

\author{
Dorota Bród \\ Rzeszow University of Technology, \\ The Faculty of Mathematics and \\ Applied Physics, \\ al. Powstańców Warszawy 12, \\ 35-959 Rzeszów, Poland \\ email: dorotab@prz.edu.pl
}

\author{
Anetta Szynal-Liana \\ Rzeszow University of Technology, \\ The Faculty of Mathematics and \\ Applied Physics, \\ al. Powstańców Warszawy 12, \\ 35-959 Rzeszów, Poland \\ email: aszynal@prz.edu.pl
}

Iwona Włoch

Rzeszow University of Technology,

The Faculty of Mathematics and Applied Physics, al. Powstańców Warszawy 12

35-959 Rzeszów, Poland

email: iwloch@prz.edu.pl

\begin{abstract}
In this paper we define dual-complex numbers with generalized Jacobsthal coefficients. We introduce one-parameter generalization of dual-complex Jacobsthal numbers - dual-complex r-Jacobsthal numbers. We investigate some algebraic properties of introduced numbers, among others Binet type formula, Catalan, Cassini, d'Ocagne and Honsberger type identities. Moreover, we present the generating function, summation formula and matrix generator for these numbers. The results are generalization of the properties for the dual-complex Jacobsthal numbers.
\end{abstract}

\section{Introduction}

The Jacobsthal sequence $\left\{J_{n}\right\}$ is one of the special cases of sequences $\left\{a_{n}\right\}$ which are defined recurrently as a linear combination of the preceding $k$ terms

2010 Mathematics Subject Classification: 11B37, 11B39

Key words and phrases: Jacobsthal numbers, dual-complex numbers, dual-complex Jacobsthal numbers, Binet formula, Catalan identity, Cassini identity 


$$
a_{n}=b_{1} a_{n-1}+b_{2} a_{n-2}+\cdots+b_{k} a_{n-k} \text { for } n \geq k,
$$

where $k \geq 2, b_{i}$ are integers, $i=1,2, \ldots, k$ and $a_{0}, a_{1}, \ldots, a_{k-1}$ are given numbers.

By recurrence (1) for $k=2$ we get (among others) definitions of the wellknown sequences:

$$
\begin{array}{llll}
\mathrm{F}_{n}=\mathrm{F}_{n-1}+\mathrm{F}_{n-2}, & \mathrm{~F}_{0}=0, & \mathrm{~F}_{1}=1 & \text { (Fibonacci numbers) } \\
\mathrm{L}_{n}=\mathrm{L}_{n-1}+\mathrm{L}_{n-2}, & \mathrm{~L}_{0}=2, & \mathrm{~L}_{1}=1 & \text { (Lucas numbers) } \\
\mathrm{J}_{n}=\mathrm{J}_{n-1}+2 \mathrm{~J}_{n-2}, & \mathrm{~J}_{0}=0, & \mathrm{~J}_{1}=1 & \text { (Jacobsthal numbers) } \\
\mathrm{P}_{n}=2 \mathrm{P}_{n-1}+\mathrm{P}_{n-2}, & \mathrm{P}_{0}=0, & \mathrm{P}_{1}=1 & \text { (Pell numbers). }
\end{array}
$$

Sequences defined by (1) are called sequences of the Fibonacci type. The first ten terms of the Jacobsthal sequence are $0,1,1,3,5,11,21,43,85,171$. This sequence is also given by formula $J_{n}=\frac{2^{n}-(-1)^{n}}{3}$, named as Binet type formula for the Jacobsthal numbers. Many authors have generalized the recurrence of the Jacobsthal sequence. In [4] a one-parameter generalization of the Jacobsthal numbers was introduced. We recall this generalization.

Let $n \geq 0, r \geq 0$ be integers. The $n$th $r$-Jacobsthal number $J(r, n)$ is defined by the following recurrence relation

$$
J(r, n)=2^{r} J(r, n-1)+\left(2^{r}+4^{r}\right) J(r, n-2) \text { for } n \geq 2
$$

with $J(r, 0)=1, J(r, 1)=1+2^{r+1}$.

For $r=0$ we have $J(0, n)=J_{n+2}$. By (2) we obtain

$$
\begin{aligned}
& J(r, 0)=1 \\
& J(r, 1)=2 \cdot 2^{r}+1 \\
& J(r, 2)=3 \cdot 4^{r}+2 \cdot 2^{r} \\
& J(r, 3)=5 \cdot 8^{r}+5 \cdot 4^{r}+2^{r} \\
& J(r, 4)=8 \cdot 16^{r}+10 \cdot 8^{r}+3 \cdot 4^{r} \\
& J(r, 5)=13 \cdot 32^{r}+20 \cdot 16^{r}+9 \cdot 8^{r}+4^{r} .
\end{aligned}
$$

In [4], it was proved that the r-Jacobsthal numbers can be used for counting of independent sets of special classes of graphs. We will recall some useful properties of the r-Jacobsthal numbers.

Theorem 1 [4] (Binet type formula) Let $\mathrm{n} \geq 0, \mathrm{r} \geq 0$ be integers. Then the $\mathrm{n}$ th $\mathrm{r}$-Jacobsthal number is given by

$$
J(r, n)=\frac{\sqrt{4 \cdot 2^{r}+5 \cdot 4^{r}}+3 \cdot 2^{r}+2}{2 \sqrt{4 \cdot 2^{r}+5 \cdot 4^{r}}} \lambda_{1}{ }^{n}+\frac{\sqrt{4 \cdot 2^{r}+5 \cdot 4^{r}}-3 \cdot 2^{r}-2}{2 \sqrt{4 \cdot 2^{r}+5 \cdot 4^{r}}} \lambda_{2}^{n},
$$


where

$$
\lambda_{1}=2^{\mathrm{r}-1}+\frac{1}{2} \sqrt{4 \cdot 2^{\mathrm{r}}+5 \cdot 4^{\mathrm{r}}}, \quad \lambda_{2}=2^{\mathrm{r}-1}-\frac{1}{2} \sqrt{4 \cdot 2^{\mathrm{r}}+5 \cdot 4^{\mathrm{r}}} .
$$

Theorem 2 [4] Let $\mathrm{n} \geq 1, \mathrm{r} \geq 0$ be integers. Then

$$
\sum_{l=0}^{n-1} J(r, l)=\frac{J(r, n)+\left(2^{r}+4^{r}\right) J(r, n-1)-2-2^{r}}{4^{r}+2^{r+1}-1} .
$$

Theorem 3 [4] (Cassini type identity) Let $\mathrm{n} \geq 1, \mathrm{r} \geq 0$ be integers. Then

$$
J(r, n+1) J(r, n-1)-J^{2}(r, n)=(-1)^{n}\left(2^{r}+1\right)^{2}\left(2^{r}+4^{r}\right)^{n-1} .
$$

Proposition 1 [4] Let $\mathrm{n} \geq 4, \mathrm{r} \geq 0$ be integers. Then

$$
J(r, n)=\left(3 \cdot 8^{r}+2 \cdot 4^{r}\right) J(r, n-3)+\left(2 \cdot 16^{r}+3 \cdot 8^{r}+4^{r}\right) J(r, n-4) .
$$

Theorem 4 [4] Let $\mathrm{n}, \mathrm{m}, \mathrm{r}$ be integers such that $\mathrm{m} \geq 2, \mathrm{n} \geq 1, \mathrm{r} \geq 0$. Then

$$
J(r, m+n)=2^{r} J(r, m-1) J(r, n)+\left(4^{r}+8^{r}\right) J(r, m-2) J(r, n-1) .
$$

Theorem 5 [4] The generating function of the sequence of $r$-Jacobsthal numbers has the following form

$$
f(x)=\frac{1+\left(1+2^{r}\right) x}{1-2^{r} x-\left(2^{r}+4^{r}\right) x^{2}} .
$$

\section{The dual-complex numbers and their properties}

The set of dual numbers is defined in the following way

$$
\mathbb{D}=\left\{\mathrm{d}: \mathrm{d}=\mathrm{u}+v \varepsilon \mid \mathrm{u}, v \in \mathbb{R}, \varepsilon^{2}=0, \quad \varepsilon \neq 0\right\} .
$$

Dual numbers were introduced by Clifford ([5]). Dual-complex numbers are known generalization of complex and dual numbers. These numbers were introduced by Majernik [8]. The set of dual-complex numbers, denoted by $\mathbb{D} \mathbb{C}$, is defined as follows

$$
\mathbb{D} \mathbb{C}=\left\{w: w=z_{1}+\varepsilon z_{2} \mid z_{1}, z_{2} \in \mathbb{C}, \varepsilon^{2}=0, \varepsilon \neq 0\right\} .
$$


If $z_{1}=x_{1}+i x_{2}$ and $z_{2}=y_{1}+i y_{2}, x_{1}, x_{2}, y_{1}, y_{2} \in \mathbb{R}$, then any dual-complex number can be written as

$$
w=x_{1}+i x_{2}+\varepsilon y_{1}+i \varepsilon y_{2} .
$$

Let $w_{1}, w_{2}$ be any dual-complex numbers, $w_{1}=z_{1}+\varepsilon z_{2}, w_{2}=z_{3}+\varepsilon z_{4}$. Addition, subtraction and multiplication of them are defined by

$$
\begin{gathered}
w_{1} \pm w_{2}=\left(z_{1} \pm z_{3}\right)+\varepsilon\left(z_{2} \pm z_{4}\right) \\
w_{1} \cdot w_{2}=z_{1} z_{3}+\varepsilon\left(z_{1} z_{4}+z_{2} z_{3}\right) .
\end{gathered}
$$

Table 1 presents multiplication scheme of dual-complex numbers.

\begin{tabular}{|c|c|c|c|c|}
$\cdot$ & 1 & $i$ & $\varepsilon$ & $i \varepsilon$ \\
\hline 1 & 1 & $i$ & $\varepsilon$ & $i \varepsilon$ \\
$i$ & $i$ & -1 & $i \varepsilon$ & $-\varepsilon$ \\
$\varepsilon$ & $\varepsilon$ & $i \varepsilon$ & 0 & 0 \\
$i \varepsilon$ & $i \varepsilon$ & $-\varepsilon$ & 0 & 0
\end{tabular}

Table 1.

Assuming that $\operatorname{Re}\left(w_{2}\right) \neq 0$, the division of two dual-complex numbers $w_{1}, w_{2}$ is given by

$$
\frac{w_{1}}{w_{2}}=\frac{z_{1}+\varepsilon z_{2}}{z_{3}+\varepsilon z_{4}}=\frac{\left(z_{1}+\varepsilon z_{2}\right)\left(z_{3}-\varepsilon z_{4}\right)}{\left(z_{3}+\varepsilon z_{4}\right)\left(z_{3}-\varepsilon z_{4}\right)}=\frac{z_{1}}{z_{3}}+\varepsilon \frac{z_{2} z_{3}-z_{1} z_{4}}{z_{3}^{2}} .
$$

The dual-complex numbers form a commutative ring with characteristics 0 . Moreover, the multiplication of these numbers gives the dual-complex numbers the structure of 2-dimensional complex Clifford algebra and 4-dimensional real Clifford algebra.

Let $w=z_{1}+\varepsilon z_{2}=x_{1}+i x_{2}+\varepsilon y_{1}+i \varepsilon y_{2}, z_{2} \neq 0$. There are five different conjugations, denoted by $w^{*}$, of dual-complex number $w$ :

$$
\begin{aligned}
& w^{* 1}=\left(z_{1}\right)^{*}+\varepsilon\left(z_{2}\right)^{*}=\left(x_{1}-i x_{2}\right)+\varepsilon\left(y_{1}-i y_{2}\right) \text { complex conjugation } \\
& w^{* 2}=z_{1}-\varepsilon z_{2}=\left(x_{1}+i x_{2}\right)-\varepsilon\left(y_{1}+i y_{2}\right) \text { dual conjugation } \\
& w^{* 3}=\left(z_{1}\right)^{*}-\varepsilon\left(z_{2}\right)^{*}=\left(x_{1}-i x_{2}\right)-\varepsilon\left(y_{1}-i y_{2}\right) \text { coupled conjugation } \\
& w^{* 4}=\left(z_{1}\right)^{*} \cdot\left(1-\varepsilon \frac{z_{2}}{z_{1}}\right)=\left(x_{1}-i x_{2}\right)\left(1-\varepsilon \frac{y_{1}+i y_{2}}{x_{1}+i x_{2}}\right) \text { dual - complex conjugation } \\
& w^{* 5}=z_{2}-\varepsilon z_{1}=\left(y_{1}+i y_{2}\right)-\varepsilon\left(x_{1}+i x_{2}\right) \text { anti }- \text { dual conjugation. }
\end{aligned}
$$


Therefore, the norms of a dual-complex number $w$ are defined as

$$
\begin{aligned}
& \mathrm{N}_{w}^{* 1}=\left\|w \cdot w^{* 1}\right\|=\sqrt{\left|z_{1}^{2}\right|+2 \varepsilon \operatorname{Re}\left(z_{1}\left(z_{2}\right)^{*}\right)} \\
& \mathrm{N}_{w}^{* 2}=\left\|w \cdot w^{* 2}\right\|=\sqrt{z_{1}^{2}} \\
& \mathrm{~N}_{w}^{* 3}=\left\|w \cdot w^{* 3}\right\|=\sqrt{\left|z_{1}^{2}\right|-2 i \varepsilon \operatorname{Im}\left(z_{1}\left(z_{2}\right)^{*}\right)} \\
& \mathrm{N}_{w}^{* 4}=\left\|w \cdot w^{* 4}\right\|=\sqrt{\left|z_{1}^{2}\right|} \\
& \mathrm{N}_{w}^{* 5}=\left\|w \cdot w^{* 5}\right\|=\sqrt{z_{1} z_{2}+\varepsilon\left(z_{2}^{2}-z_{1}^{2}\right)} .
\end{aligned}
$$

In the literature there are a lot of studies about numbers of the Fibonacci type. Many authors investigated quaternions, split quaternions, hyperbolic numbers, dual-hyperbolic numbers and dual-complex numbers with Fibonacci,

Lucas, Pell, Jacobsthal coefficients, see [1, 2, 7, 9, 10]. In [6] dual-complex Fibonacci and Lucas numbers were studied. In [3] many interesting properties of dual-complex k-Pell quaternions were given. In this paper we introduce dual-complex numbers with generalized Jacobsthal numbers coefficients. We use one-parameter generalization of the Jacobsthal numbers - r-Jacobsthal numbers.

\section{The dual-complex r-Jacobsthal numbers}

For nonnegative integers $n$ and $r$ the $n$th dual-complex $r$-Jacobsthal number $\mathbb{D C J}(r, n)$ is defined as

$$
\mathbb{D} \mathbb{C} J(r, n)=J(r, n)+i J(r, n+1)+\varepsilon J(r, n+2)+i \varepsilon J(r, n+3),
$$

where $J(r, n)$ is given by $(2)$.

Note that for $r=0$ we obtain $\mathbb{D} \mathbb{C}(0, n)=\mathbb{D} \mathbb{C} J_{n+2}$, where $\mathbb{D} \mathbb{C} J_{n}$ denotes the $n$th dual-complex Jacobsthal number.

Now we give five conjugations of dual-complex r-Jacobsthal numbers 1) complex conjugation

$$
\mathbb{D C J}(r, n)^{* 1}=J(r, n)-i J(r, n+1)+\varepsilon J(r, n+2)-i \varepsilon J(r, n+3),
$$

2) dual conjugation

$$
\mathbb{D C} J(r, n)^{* 2}=J(r, n)+i J(r, n+1)-\varepsilon J(r, n+2)-i \varepsilon J(r, n+3),
$$


3) coupled conjugation

$$
\mathbb{D C J}(r, n)^{* 3}=J(r, n)-i J(r, n+1)-\varepsilon J(r, n+2)+i \varepsilon J(r, n+3),
$$

4) dual-complex conjugation

$$
\mathbb{D C J}(r, n)^{* 4}=(J(r, n)-i J(r, n+1))\left(1-\varepsilon \frac{J(r, n+2)+i J(r, n+3)}{J(r, n)+i J(r, n+1)}\right),
$$

5) anti-dual conjugation

$$
\mathbb{D C} J(r, n)^{* 5}=J(r, n+2)+i J(r, n+3)-\varepsilon J(r, n)-i \varepsilon J(r, n+1) .
$$

By simple calculations we can give the following relations

$$
\begin{aligned}
& \mathbb{D} \mathbb{C J}(r, n) \cdot \mathbb{D} \mathbb{C J}(r, n)^{* 1}=J^{2}(r, n)+J^{2}(r, n+1)+2 \varepsilon[J(r, n) J(r, n+2) \\
& +J(r, n+1) J(r, n+3)] \text {, } \\
& \mathbb{D} \mathbb{C J}(r, n) \cdot \mathbb{D C J}(r, n)^{* 2}=J^{2}(r, n)-J^{2}(r, n+1)+2 i J(r, n) J(r, n+1), \\
& \mathbb{D C J}(r, n) \cdot \mathbb{D C J}(r, n)^{* 3}=J^{2}(r, n)+J^{2}(r, n+1)+2 i \varepsilon[J(r, n) J(r, n+3) \\
& -J(r, n+1) J(r, n+2)] \text {, } \\
& \mathbb{D} \mathbb{C J}(r, n) \cdot \mathbb{D C J}(r, n)^{* 4}=J^{2}(r, n)+J^{2}(r, n+1), \\
& \mathbb{D C J}(r, n) \cdot \mathbb{D C J}(r, n)^{* 5}=J(r, n) J(r, n+2)-J(r, n+1) J(r, n+3) \\
& +\mathfrak{i}[J(r, n) J(r, n+3)+J(r, n+1) J(r, n+2)] \\
& +\varepsilon\left[-J^{2}(r, n)+J^{2}(r, n+1)\right. \\
& \left.+J^{2}(r, n+2)-J^{2}(r, n+3)\right] \\
& +2 i \varepsilon(J(r, n+2) J(r, n+3)-J(r, n) J(r, n+1)), \\
& \mathbb{D} \mathbb{C J}(r, n)+\mathbb{D} \mathbb{C} J(r, n)^{* 1}=2[J(r, n)+\varepsilon J(r, n+2)], \\
& \mathbb{D} \mathbb{C J}(r, n)+\mathbb{D} \mathbb{C J}(r, n)^{* 2}=2[J(r, n)+i J(r, n+1)] \text {, } \\
& \mathbb{D} \mathbb{C J}(r, n)+\mathbb{D} \mathbb{C J}(r, n)^{* 3}=2[J(r, n)+i \varepsilon J(r, n+3)], \\
& \mathbb{D} \mathbb{C J}(r, n)-\varepsilon \mathbb{D} \mathbb{C} J(r, n)^{* 5}=J(r, n)+i J(r, n+1), \\
& \varepsilon \mathbb{D} \mathbb{C} J(r, n)+\mathbb{D} \mathbb{C J}(r, n)^{* 5}=J(r, n+2)+i J(r, n+3) \text {. }
\end{aligned}
$$

Using the definition of the dual-complex $r$-Jacobsthal number we get the following recurrence relations.

Proposition 2 Let $\mathrm{n} \geq 0, \mathrm{r} \geq 0$ be integers. Then

$$
\mathbb{D} \mathbb{C J}(r, n+2)=2^{r} \mathbb{D} \mathbb{C} J(r, n+1)+\left(2^{r}+4^{r}\right) \mathbb{D} \mathbb{C J}(r, n)
$$

with

$$
\begin{aligned}
\mathbb{D C J}(r, 0)= & 1+i\left(2^{r+1}+1\right)+\varepsilon\left(3 \cdot 4^{r}+2^{r+1}\right)+i \varepsilon\left(5 \cdot 8^{r}+5 \cdot 4^{r}+2^{r}\right), \\
\mathbb{D C J}(r, 1)= & 2^{r+1}+1+i\left(3 \cdot 4^{r}+2^{r+1}\right)+\varepsilon\left(5 \cdot 8^{r}+5 \cdot 4^{r}+2^{r}\right) \\
& +i \varepsilon\left(8 \cdot 16^{r}+10 \cdot 8^{r}+3 \cdot 4^{r}\right) .
\end{aligned}
$$


Proof. By formulas (4) and (2) we get

$$
\begin{aligned}
2^{r} \mathbb{D} \mathbb{C J}(r, n+1)+\left(2^{r}+4^{r}\right) \mathbb{D} \mathbb{C} J(r, n) \\
=2^{r}(J(r, n+1)+i J(r, n+2)+\varepsilon J(r, n+3)+i \varepsilon J(r, n+4)) \\
\quad+\left(2^{r}+4^{r}\right)(J(r, n)+i J(r, n+1)+\varepsilon J(r, n+2)+i \varepsilon J(r, n+3)) \\
=J(r, n+2)+i J(r, n+3)+\varepsilon J(r, n+4)+i \varepsilon J(r, n+5)=\mathbb{D} \mathbb{C} J(r, n+2) .
\end{aligned}
$$

Proposition 3 Let $\mathrm{n} \geq 4, \mathrm{r} \geq 0$ be integers. Then

$\mathbb{D} \mathbb{C J}(r, n)=\left(3 \cdot 8^{r}+2 \cdot 4^{r}\right) \mathbb{D} \mathbb{C} J(r, n-3)+\left(2 \cdot 16^{r}+3 \cdot 8^{r}+4^{r}\right) \mathbb{D} \mathbb{C} J(r, n-4)$.

Proof. Let $A=3 \cdot 8^{r}+2 \cdot 4^{r}, B=2 \cdot 16^{r}+3 \cdot 8^{r}+4^{r}$. By Proposition 1 we have

$$
\begin{aligned}
\mathbb{D C J}(r, n)= & J(r, n)+i J(r, n+1)+\varepsilon J(r, n+2)+i \varepsilon J(r, n+3) \\
= & A \cdot J(r, n-3)+B \cdot J(r, n-4)+i(A \cdot J(r, n-2)+B \cdot J(r, n-3)) \\
& +\varepsilon(A \cdot J(r, n-1)+B \cdot J(r, n-2))+i \varepsilon(A \cdot J(r, n)+B \cdot J(r, n-1)) \\
= & A(J(r, n-3)+i J(r, n-2)+\varepsilon J(r, n-1)+i \varepsilon J(r, n)) \\
& +B(J(r, n-4)+i J(r, n-3)+\varepsilon J(r, n-2)+i \varepsilon J(r, n-1)) .
\end{aligned}
$$

Hence we get

$\mathbb{D} \mathbb{C} J(r, n)=A \cdot \mathbb{D} \mathbb{C} J(r, n-3)+B \cdot \mathbb{D} \mathbb{C} J(r, n-4)$.

Theorem 6 Let $\mathrm{n} \geq 0, \mathrm{r} \geq 0$ be integers. Then

$$
\begin{aligned}
& \mathbb{D} \mathbb{C J}(r, n)-i \mathbb{D} \mathbb{C} J(r, n+1)-\varepsilon \mathbb{D} \mathbb{C} J(r, n+2)+i \varepsilon \mathbb{D} \mathbb{C} J(r, n+3)= \\
& =J(r, n)+J(r, n+2) .
\end{aligned}
$$

Proof. By simple calculations we get

$$
\begin{aligned}
& \mathbb{D} \mathbb{C} J(r, n)-i \mathbb{D C J}(r, n+1)-\varepsilon \mathbb{D} \mathbb{C} J(r, n+2)+i \varepsilon \mathbb{D} \mathbb{C} J(r, n+3)= \\
&=J(r, n)+i J(r, n+1)+\varepsilon J(r, n+2)+i \varepsilon J(r, n+3) \\
&-i(J(r, n+1)+i J(r, n+2)+\varepsilon J(r, n+3)+i \varepsilon J(r, n+4)) \\
&-\varepsilon(J(r, n+2)+i J(r, n+3)+\varepsilon J(r, n+4)+i \varepsilon J(r, n+5)) \\
&+i \varepsilon(J(r, n+3)+i J(r, n+4)+\varepsilon J(r, n+5)+i \varepsilon J(r, n+6)) \\
&= J(r, n)+i J(r, n+1)+\varepsilon J(r, n+2)+i \varepsilon J(r, n+3) \\
&-i J(r, n+1)+J(r, n+2)-i \varepsilon J(r, n+3)+\varepsilon J(r, n+4) \\
&-\varepsilon J(r, n+2)-i \varepsilon J(r, n+3)+i \varepsilon J(r, n+3)-\varepsilon J(r, n+4) \\
&= J(r, n)+J(r, n+2),
\end{aligned}
$$


which ends the proof.

In the next theorem we present the Binet type formula for the dual-complex r-Jacobsthal numbers.

Theorem 7 Let $\mathrm{n} \geq 0, \mathrm{r} \geq 0$ be integers. Then the $\mathrm{n}$ th dual-complex $\mathrm{r}$ Jacobsthal number is given by

$$
\mathbb{D C J}(r, n)=C_{1} \widehat{\lambda_{1}} \lambda_{1}^{n}+C_{2} \widehat{\lambda_{2}} \lambda_{2}^{n},
$$

where

$$
\begin{array}{ll}
\lambda_{1}=2^{\mathrm{r}-1}+\frac{1}{2} \sqrt{4 \cdot 2^{\mathrm{r}}+5 \cdot 4^{\mathrm{r}}}, & \lambda_{2}=2^{\mathrm{r}-1}-\frac{1}{2} \sqrt{4 \cdot 2^{\mathrm{r}}+5 \cdot 4^{\mathrm{r}}}, \\
\widehat{\lambda_{1}}=1+i \lambda_{1}+\varepsilon \lambda_{1}^{2}+i \varepsilon \lambda_{1}^{3}, & \widehat{\lambda_{2}}=1+i \lambda_{2}+\varepsilon \lambda_{2}^{2}+i \varepsilon \lambda_{2}^{3}, \\
\mathrm{C}_{1}=\frac{\sqrt{4 \cdot 2^{\mathrm{r}}+5 \cdot 4^{\mathrm{r}}}+3 \cdot 2^{\mathrm{r}}+2}{2 \sqrt{4 \cdot 2^{\mathrm{r}}+5 \cdot 4^{\mathrm{r}}}}, & \mathrm{C}_{2}=\frac{\sqrt{4 \cdot 2^{\mathrm{r}}+5 \cdot 4^{\mathrm{r}}}-3 \cdot 2^{\mathrm{r}}-2}{2 \sqrt{4 \cdot 2^{\mathrm{r}}+5 \cdot 4^{\mathrm{r}}}} .
\end{array}
$$

Proof. By Theorem 1 we get

$$
\begin{aligned}
\mathbb{D C J}(r, n)= & J(r, n)+i J(r, n+1)+\varepsilon J(r, n+2)+i \varepsilon J(r, n+3) \\
= & C_{1} \lambda_{1}^{n}+C_{2} \lambda_{2}^{n}+i\left(C_{1} \lambda_{1}^{n+1}+C_{2} \lambda_{2}^{n+1}\right) \\
& +\varepsilon\left(C_{1} \lambda_{1}^{n+2}+C_{2} \lambda_{2}^{n+2}\right)+i \varepsilon\left(C_{1} \lambda_{1}^{n+3}+C_{2} \lambda_{2}^{n+3}\right) \\
= & C_{1} \lambda_{1}^{n}\left(1+i \lambda_{1}+\varepsilon \lambda_{1}^{2}+i \varepsilon \lambda_{1}^{3}\right)+C_{2} \lambda_{2}^{n}\left(1+i \lambda_{2}+\varepsilon \lambda_{2}^{2}+i \varepsilon \lambda_{2}^{3}\right) \\
= & C_{1} \hat{\lambda_{1}} \lambda_{1}^{n}+C_{2} \hat{\lambda}_{2} \lambda_{2}^{n},
\end{aligned}
$$

which ends the proof.

Corollary 1 (Binet type formula for dual-complex Jacobsthal numbers) Let $\mathrm{n} \geq 0$ be an integer. Then

$$
\mathbb{D C} J_{n}=\frac{1}{3}\left[2^{\mathfrak{n}}(1+2 i+4 \varepsilon+8 i \varepsilon)-(-1)^{\mathrm{n}}(1-i+\varepsilon-i \varepsilon)\right] .
$$

Proof. By formula (5), for $r=0$ we obtain $\lambda_{1}=2, \lambda_{2}=-1, C_{1}=\frac{4}{3}, C_{2}=-\frac{1}{3}$. Moreover,

$$
\begin{aligned}
\mathbb{D C J}(0, \mathrm{n}) & =\frac{4}{3} \cdot 2^{\mathfrak{n}}(1+2 i+4 \varepsilon+8 i \varepsilon)-\frac{1}{3}(-1)^{\mathrm{n}}(1-i+\varepsilon-i \varepsilon) \\
& =\frac{1}{3} \cdot 2^{\mathrm{n}+2}(1+2 i+4 \varepsilon+8 i \varepsilon)-\frac{1}{3}(-1)^{\mathrm{n}+2}(1-i+\varepsilon-i \varepsilon) \\
& =\mathbb{D} \mathbb{C} J_{n+2} .
\end{aligned}
$$




\section{Some identities involving the dual-complex r-Jacobsthal numbers}

In this section we give some identities such as Catalan, Cassini and d'Ocagne type identities for the dual-complex $r$-Jacobsthal numbers. These identities can be proved by using formula (5). We will need the following lemma.

Lemma 1 Let $\widehat{\lambda_{1}}=1+i \lambda_{1}+\varepsilon \lambda_{1}^{2}+i \varepsilon \lambda_{1}^{3}, \hat{\lambda_{2}}=1+i \lambda_{2}+\varepsilon \lambda_{2}^{2}+i \varepsilon \lambda_{2}^{3}$, where

$$
\lambda_{1}=2^{\mathrm{r}-1}+\frac{1}{2} \sqrt{4 \cdot 2^{\mathrm{r}}+5 \cdot 4^{\mathrm{r}}}, \quad \lambda_{2}=2^{\mathrm{r}-1}-\frac{1}{2} \sqrt{4 \cdot 2^{\mathrm{r}}+5 \cdot 4^{\mathrm{r}}} .
$$

Then

$$
\begin{aligned}
\widehat{\lambda_{1}} \widehat{\lambda_{2}}=\widehat{\lambda_{2}} \hat{\lambda_{1}=} & 1+4^{r}+2^{r}+2^{r} i+\left(2^{r+1}+5 \cdot 4^{r}+5 \cdot 8^{r}+3 \cdot 16^{r}\right) \mathcal{E} \\
& +\left(3 \cdot 8^{r}+2 \cdot 4^{r}\right) i \varepsilon .
\end{aligned}
$$

Proof. By simple calculations we get

$$
\begin{aligned}
\widehat{\lambda_{1}} \widehat{\lambda_{2}=} & 1+i \lambda_{2}+\varepsilon \lambda_{2}^{2}+i \varepsilon \lambda_{2}^{3}+i \lambda_{1}-\lambda_{1} \lambda_{2}+i \varepsilon \lambda_{1} \lambda_{2}^{2} \\
& -\varepsilon \lambda_{1} \lambda_{2}^{3}+\varepsilon \lambda_{1}^{2}+i \varepsilon \lambda_{1}^{2} \lambda_{2}+i \varepsilon \lambda_{1}^{3}-\varepsilon \lambda_{1}^{3} \lambda_{2} \\
= & 1-\lambda_{1} \lambda_{2}+\left(\lambda_{1}+\lambda_{2}\right) i+\left(\lambda_{1}^{2}+\lambda_{2}^{2}\right)\left(1-\lambda_{1} \lambda_{2}\right) \varepsilon \\
& +\left(\lambda_{1}^{3}+\lambda_{2}^{3}+\lambda_{1} \lambda_{2}\left(\lambda_{1}+\lambda_{2}\right)\right) i \varepsilon .
\end{aligned}
$$

Using the equalities

$$
\begin{aligned}
\lambda_{1} \lambda_{2} & =-\left(4^{\mathrm{r}}+2^{\mathrm{r}}\right), \\
\lambda_{1}+\lambda_{2} & =2^{\mathrm{r}}, \\
\lambda_{1}^{2}+\lambda_{2}^{2} & =\left(\lambda_{1}+\lambda_{2}\right)^{2}-2 \lambda_{1} \lambda_{2}=3 \cdot 4^{\mathrm{r}}+2^{\mathrm{r}+1}, \\
\lambda_{1}^{3}+\lambda_{2}^{3} & =\left(\lambda_{1}+\lambda_{2}\right)^{3}-3 \lambda_{1} \lambda_{2}\left(\lambda_{1}+\lambda_{2}\right)=4 \cdot 8^{\mathrm{r}}+3 \cdot 4^{\mathrm{r}},
\end{aligned}
$$

we get the result.

Theorem 8 (Catalan type identity for dual-complex $\mathrm{r}$-Jacobsthal numbers) Let $\mathrm{n} \geq 0, \mathrm{~m} \geq 0, \mathrm{r} \geq 0$ be integers such that $\mathrm{n} \geq \mathrm{m}$. Then

$$
\begin{aligned}
& (\mathbb{D C J}(r, n))^{2}-\mathbb{D C} J(r, n-m) \cdot \mathbb{D} \mathbb{C J}(r, n+m)= \\
& \quad=-\frac{\left(-4^{r}-2^{r}\right)^{n}\left(1+2^{r}\right)^{2}}{4 \cdot 2^{r}+5 \cdot 4^{r}}\left(2-\left(\frac{\lambda_{1}}{\lambda_{2}}\right)^{m}-\left(\frac{\lambda_{2}}{\lambda_{1}}\right)^{m}\right) \widehat{\lambda_{1}} \widehat{\lambda_{2}},
\end{aligned}
$$

where $\widehat{\lambda_{1}} \widehat{\lambda_{2}}$ is given by $(6)$. 
Proof. By formula (5) we get

$$
\begin{aligned}
& (\mathbb{D} \mathbb{C J}(r, n))^{2}-\mathbb{D} \mathbb{C J}(r, n-m) \cdot \mathbb{D C J}(r, n+m) \\
& =\left(C_{1} \hat{\lambda_{1}} \lambda_{1}^{n}+C_{2} \hat{\lambda_{2}} \lambda_{2}^{n}\right)\left(C_{1} \hat{\lambda_{1}} \lambda_{1}^{n}+C_{2} \hat{\lambda_{2}} \lambda_{2}^{n}\right) \\
& -\left(C_{1} \hat{\lambda_{1}} \lambda_{1}^{n-m}+C_{2} \hat{\lambda_{2}} \lambda_{2}^{n-m}\right)\left(C_{1} \hat{\lambda_{1}} \lambda_{1}^{n+m}+C_{2} \hat{\lambda_{2}} \lambda_{2}^{n+m}\right) \\
& =2 \mathrm{C}_{1} \mathrm{C}_{2} \hat{\lambda_{1}} \widehat{\lambda_{2}}\left(\lambda_{1} \lambda_{2}\right)^{\mathrm{n}}-\mathrm{C}_{1} \mathrm{C}_{2} \hat{\lambda_{1}} \widehat{\lambda_{2}} \lambda_{1}^{n+m} \lambda_{2}^{n-m}-\mathrm{C}_{1} \mathrm{C}_{2} \hat{\lambda_{1}} \widehat{\lambda_{2}} \lambda_{1}^{n-m} \lambda_{2}^{n+m} \\
& =C_{1} C_{2} \hat{\lambda_{1}} \widehat{\lambda_{2}}\left(\lambda_{1} \lambda_{2}\right)^{n}\left(2-\left(\frac{\lambda_{1}}{\lambda_{2}}\right)^{m}-\left(\frac{\lambda_{2}}{\lambda_{1}}\right)^{m}\right) \text {. }
\end{aligned}
$$

Since $\lambda_{1} \lambda_{2}=-\left(4^{r}+2^{r}\right)$ and $C_{1} C_{2}=-\frac{\left(1+2^{r}\right)^{2}}{4 \cdot 2^{r}+5 \cdot 4^{r}}$, we have

$$
\begin{aligned}
& (\mathbb{D} \mathbb{C J}(r, n))^{2}-\mathbb{D} \mathbb{C J}(r, n-m) \cdot \mathbb{D C J}(r, n+m)= \\
& =C_{1} C_{2}\left(-4^{r}-2^{r}\right)^{n} \hat{\lambda_{1}} \widehat{\lambda_{2}}\left(2-\left(\frac{\lambda_{1}}{\lambda_{2}}\right)^{m}-\left(\frac{\lambda_{2}}{\lambda_{1}}\right)^{\mathrm{m}}\right) \\
& =-\frac{\left(-4^{r}-2^{r}\right)^{n}\left(1+2^{r}\right)^{2}}{4 \cdot 2^{r}+5 \cdot 4^{r}}\left(2-\left(\frac{\lambda_{1}}{\lambda_{2}}\right)^{m}-\left(\frac{\lambda_{2}}{\lambda_{1}}\right)^{m}\right) \hat{\lambda_{1}} \widehat{\lambda_{2}},
\end{aligned}
$$

which ends the proof.

Corollary 2 (Cassini type identity for dual-complex $\mathrm{r}$-Jacobsthal numbers) Let $\mathrm{n} \geq 1, \mathrm{r} \geq 0$ be integers. Then

$$
(\mathbb{D C J}(r, n))^{2}-\mathbb{D} \mathbb{C J}(r, n-1) \cdot \mathbb{D} \mathbb{C J}(r, n+1)=\left(-4^{r}-2^{r}\right)^{n-1}\left(1+2^{r}\right)^{2} \hat{\lambda_{1}} \widehat{\lambda_{2}} .
$$

In particular, by Theorem 8, we obtain the following formulas for the dualcomplex Jacobsthal numbers.

Corollary 3 (Catalan type identity for dual-complex Jacobsthal numbers) Let $\mathrm{n} \geq 0, \mathrm{~m} \geq 0$, be integers such that $\mathrm{n} \geq \mathrm{m}$. Then

$$
\left.\left(\mathbb{D C} J_{n}\right)^{2}-\mathbb{D C} J_{n-m} \cdot \mathbb{D C} J_{n+m}=\frac{4}{9}(-2)^{n-m}\left((-2)^{m}-1\right)\right)^{2}(3+i+15 \varepsilon+5 i \varepsilon) .
$$

Corollary 4 (Cassini type identity for dual-complex Jacobsthal numbers) Let $\mathrm{n} \geq 1$ be an integer. Then

$$
\left(\mathbb{D C} J_{n}\right)^{2}-\mathbb{D C} J_{n-1} \cdot \mathbb{D} \mathbb{C} J_{n+1}=4(-2)^{n-1}(3+i+15 \varepsilon+5 i \varepsilon) .
$$


Theorem 9 (Vajda type identity for dual-complex $\mathrm{r}$-Jacobsthal numbers) Let $\mathrm{n} \geq 0, \mathrm{~m} \geq 0, \mathrm{k} \geq 0, \mathrm{r} \geq 0$ be integers such that $\mathrm{n} \geq \mathrm{k}$. Then

$$
\begin{aligned}
& \mathbb{D} \mathbb{C J}(r, m+k) \cdot \mathbb{D} \mathbb{C} J(r, n-k)-\mathbb{D} \mathbb{C} J(r, m) \cdot \mathbb{D} \mathbb{C J}(r, n)= \\
& \quad=-\frac{\left(1+2^{r}\right)^{2}\left(-4^{r}-2^{r}\right)^{m}}{4 \cdot 2^{r}+5 \cdot 4^{r}}\left(\lambda_{2}^{n-m}\left[\left(\frac{\lambda_{1}}{\lambda_{2}}\right)^{k}-1\right]+\lambda_{1}^{n-m}\left[\left(\frac{\lambda_{2}}{\lambda_{1}}\right)^{k}-1\right]\right) \hat{\lambda_{1}} \widehat{\lambda_{2}},
\end{aligned}
$$

where $\widehat{\lambda_{1}} \widehat{\lambda_{2}}$ is given by (6).

Proof. By (5) we get

$$
\begin{aligned}
& \mathbb{D} \mathbb{C J}(r, m+k) \cdot \mathbb{D} \mathbb{C} J(r, n-k)-\mathbb{D} \mathbb{C}(r, m) \cdot \mathbb{D} \mathbb{C} J(r, n)= \\
& =\left(C_{1} \widehat{\lambda_{1}} \lambda_{1}^{m+k}+C_{2} \widehat{\lambda_{2}} \lambda_{2}^{m+k}\right)\left(C_{1} \widehat{\lambda_{1}} \lambda_{1}^{n-k}+C_{2} \widehat{\lambda_{2}} \lambda_{2}^{n-k}\right) \\
& -\left(\mathrm{C}_{1} \hat{\lambda_{1}} \lambda_{1}^{\mathrm{m}}+\mathrm{C}_{2} \hat{\lambda}_{2} \lambda_{2}^{\mathrm{m}}\right)\left(\mathrm{C}_{1} \hat{\lambda}_{1} \lambda_{1}^{\mathrm{n}}+\mathrm{C}_{2} \hat{\lambda_{2}} \lambda_{2}^{\mathrm{n}}\right) \\
& =\mathrm{C}_{1} \mathrm{C}_{2} \widehat{\lambda_{1}} \hat{\lambda_{2}}\left(\lambda_{1}^{\mathrm{m}+\mathrm{k}} \lambda_{2}^{\mathrm{n}-\mathrm{k}}+\lambda_{1}^{\mathrm{n}-\mathrm{k}} \lambda_{2}^{\mathrm{m}+\mathrm{k}}-\lambda_{1}^{\mathrm{m}} \lambda_{2}^{\mathrm{n}}-\lambda_{1}^{n} \lambda_{2}^{\mathrm{m}}\right) \\
& =\mathrm{C}_{1} \mathrm{C}_{2} \hat{\lambda_{1}}{\hat{\lambda_{2}}}_{2}\left(\lambda_{1} \lambda_{2}\right)^{\mathrm{m}}\left(\lambda_{2}^{\mathrm{n}-\mathrm{m}}\left[\left(\frac{\lambda_{1}}{\lambda_{2}}\right)^{\mathrm{k}}-1\right]+\lambda_{1}^{\mathrm{n}-\mathrm{m}}\left[\left(\frac{\lambda_{2}}{\lambda_{1}}\right)^{\mathrm{k}}-1\right]\right) \\
& =-\frac{\left(1+2^{r}\right)^{2}\left(-4^{r}-2^{r}\right)^{m}}{4 \cdot 2^{r}+5 \cdot 4^{r}}\left(\lambda_{2}^{n-m}\left[\left(\frac{\lambda_{1}}{\lambda_{2}}\right)^{k}-1\right]+\lambda_{1}^{n-m}\left[\left(\frac{\lambda_{2}}{\lambda_{1}}\right)^{k}-1\right]\right) \widehat{\lambda_{1}} \widehat{\lambda_{2}} \text {. }
\end{aligned}
$$

Theorem 10 (Vajda type identity for dual-complex Jacobsthal numbers) Let $\mathrm{n} \geq 0, \mathrm{~m} \geq 0, \mathrm{k} \geq 0$ be integers such that $\mathrm{n} \geq \mathrm{k}$. Then

$$
\begin{aligned}
& \mathbb{D} \mathbb{C} J_{m+k} \cdot \mathbb{D} \mathbb{C} J_{n-k}-\mathbb{D} \mathbb{C} J_{m} \cdot \mathbb{D} \mathbb{C} J_{n}= \\
& \quad=-\frac{4}{9}(-2)^{m}\left((-1)^{n-m}\left[(-2)^{k}-1\right]+2^{n-m}\left[\left(-\frac{1}{2}\right)^{k}-1\right]\right)(3+i+15 \varepsilon+5 i \varepsilon) .
\end{aligned}
$$

Theorem 11 (d'Ocagne type identity for dual-complex $r$-Jacobsthal numbers) Let $\mathrm{n} \geq 0, \mathrm{~m} \geq 0, \mathrm{r} \geq 0$ be integers such that $\mathrm{n} \geq \mathrm{m}$. Then

$$
\begin{aligned}
& \mathbb{D} \mathbb{C J}(r, n) \cdot \mathbb{D} \mathbb{C} J(r, m+1)-\mathbb{D} \mathbb{C J}(r, n+1) \cdot \mathbb{D} \mathbb{C J}(r, m)= \\
& =\frac{\left(1+2^{r}\right)^{2} \sqrt{4 \cdot 2^{r}+5 \cdot 4^{r}}}{4 \cdot 2^{r}+5 \cdot 4^{r}}\left(-4^{r}-2^{r}\right)^{m}\left(\lambda_{1}^{n-m}-\lambda_{2}^{n-m}\right) \hat{\lambda_{1}} \widehat{\lambda_{2}},
\end{aligned}
$$

where $\widehat{\lambda_{1}} \widehat{\lambda_{2}}$ is given by (6). 
Proof. Using the Binet type formula (5) we get

$$
\begin{aligned}
& \mathbb{D} \mathbb{C J}(r, n) \cdot \mathbb{D C J}(r, m+1)-\mathbb{D C} J(r, n+1) \cdot \mathbb{D C} J(r, m)= \\
&=\left(C_{1} \widehat{\lambda_{1}} \lambda_{1}^{n}+C_{2} \widehat{\lambda_{2}} \lambda_{2}^{n}\right)\left(C_{1} \widehat{\lambda_{1}} \lambda_{1}^{m+1}+C_{2} \widehat{\lambda_{2}} \lambda_{2}^{m+1}\right) \\
&-\left(C_{1} \widehat{\lambda_{1}} \lambda_{1}^{n+1}+C_{2} \widehat{\lambda_{2}} \lambda_{2}^{n+1}\right)\left(C_{1} \widehat{\lambda_{1}} \lambda_{1}^{m}+C_{2} \widehat{\lambda_{2}} \lambda_{2}^{m}\right) \\
&= C_{1} C_{2} \widehat{\lambda_{1}} \widehat{\lambda_{2}}\left(\lambda_{1}^{m+1} \lambda_{2}^{n}+\lambda_{1}^{n} \lambda_{2}^{m+1}-\lambda_{1}^{m} \lambda_{2}^{n+1}-\lambda_{1}^{n+1} \lambda_{2}^{m}\right) \\
&= C_{1} C_{2} \widehat{\lambda_{1}} \widehat{\lambda_{2}}\left(\lambda_{1} \lambda_{2}\right)^{m}\left(\lambda_{1} \lambda_{2}^{n-m}+\lambda_{1}^{n-m} \lambda_{2}-\lambda_{2}^{n-m+1}-\lambda_{1}^{n-m+1}\right) \\
&= C_{1} C_{2}\left(\lambda_{2}-\lambda_{1}\right)\left(\lambda_{1} \lambda_{2}\right)^{m} \widehat{\lambda_{1}} \widehat{\lambda_{2}}\left(\lambda_{1}^{n-m}-\lambda_{2}^{n-m}\right) \\
&= \frac{\left(1+2^{r}\right)^{2} \sqrt{4 \cdot 2^{r}+5 \cdot 4^{r}}}{4 \cdot 2^{r}+5 \cdot 4^{r}}\left(-4^{r}-2^{r}\right)^{m}\left(\lambda_{1}^{n-m}-\lambda_{2}^{n-m}\right) \widehat{\lambda_{1}} \widehat{\lambda_{2}} .
\end{aligned}
$$

Corollary 5 (d'Ocagne type identity for dual-complex Jacobsthal numbers) Let $\mathrm{n} \geq 0, \mathrm{~m} \geq 0$ be integers such that $\mathrm{n} \geq \mathrm{m}$. Then

$$
\begin{aligned}
& \mathbb{D C} J_{n} \cdot \mathbb{D} \mathbb{C} J_{m+1}-\mathbb{D} \mathbb{C} J_{n+1} \cdot \mathbb{D C} J_{m}= \\
& \quad=\frac{4}{3}(-2)^{m}\left(2^{n-m}-(-1)^{n-m}\right)(3+i+15 \varepsilon+5 i \varepsilon) .
\end{aligned}
$$

Now we give a summation formula for the dual-complex r-Jacobsthal numbers.

Theorem 12 Let $\mathrm{n} \geq 1, \mathrm{r} \geq 0$ be integers. Then

$$
\begin{aligned}
\sum_{l=0}^{n} \mathbb{D} \mathbb{C J}(r, l)= & \frac{1}{4^{r}+2^{r+1}-1}\left[\mathbb{D} \mathbb{C} J(r, n+1)+\left(2^{r}+4^{r}\right) \mathbb{D} \mathbb{C} J(r, n)\right. \\
& \left.-(1+i+\varepsilon+i \varepsilon)\left(2+2^{r}\right)\right] \\
& -i-\left(2+2^{r+1}\right) \varepsilon-\left(2^{r+2}+3 \cdot 4^{r}+2\right) i \varepsilon .
\end{aligned}
$$

Proof. By formula (3) we have

$$
\begin{aligned}
\sum_{l=0}^{n} \mathbb{D C J}(r, l) & =\sum_{l=0}^{n}(J(r, l)+i J(r, l+1)+\varepsilon J(r, l+2)+i \varepsilon J(r, l+3)) \\
& =\sum_{l=0}^{n} J(r, l)+\sum_{l=0}^{n} i J(r, l+1)+\sum_{l=0}^{n} \varepsilon J(r, l+2)+\sum_{l=0}^{n} i \varepsilon J(r, l+3) \\
& =\frac{1}{4^{r}+2^{r+1}-1}\left[J(r, n+1)+\left(2^{r}+4^{r}\right) J(r, n)-2-2^{r}\right.
\end{aligned}
$$


On a new one-parameter generalization of dual-complex Jacobsthal numbers 139

$$
\begin{aligned}
& +i\left(J(r, n+2)+\left(2^{r}+4^{r}\right) J(r, n+1)-2-2^{r}\right) \\
& +\varepsilon\left(J(r, n+3)+\left(2^{r}+4^{r}\right) J(r, n+2)-2-2^{r}\right) \\
& \left.+i \varepsilon\left(J(r, n+4)+\left(2^{r}+4^{r}\right) J(r, n+3)-2-2^{r}\right)\right] \\
& -i J(r, 0)-\varepsilon(J(r, 0)+J(r, 1))-i \varepsilon(J(r, 0)+J(r, 1)+J(r, 2)) .
\end{aligned}
$$

By simple calculations we get

$$
\begin{aligned}
\sum_{l=0}^{n} \mathbb{D} \mathbb{C} J(r, l)= & \frac{1}{4^{r}+2^{r+1}-1}[J(r, n+1)+i J(r, n+2) \\
& +\varepsilon J(r, n+3)+i \varepsilon J(r, n+4) \\
& +\left(2^{r}+4^{r}\right)(J(r, n)+i J(r, n+1)+\varepsilon J(r, n+2)+i \varepsilon J(r, n+3)) \\
& \left.-\left(2+2^{r}\right)(1+i+\varepsilon+i \varepsilon)\right]-i-\left(2^{r+1}+2\right) \varepsilon-\left(2^{r+2}+3 \cdot 4^{r}+2\right) i \varepsilon \\
= & \frac{\mathbb{D} J(r, n+1)+\left(2^{r}+4^{r}\right) \mathbb{D} \mathbb{C} J(r, n)-(1+i+\varepsilon+i \varepsilon)\left(2+2^{r}\right)}{4^{r}+2^{r+1}-1} \\
& -i-\left(2+2^{r+1}\right) \varepsilon-\left(2^{r+2}+3 \cdot 4^{r}+2\right) i \varepsilon .
\end{aligned}
$$

In particular, we obtain the following formula for the dual-complex Jacobsthal numbers.

Corollary 6 Let $\mathrm{n} \geq 1$ be an integer. Then

$$
\sum_{l=0}^{n} \mathbb{D C} J_{l}=\frac{\mathbb{D C} J_{n+2}-\mathbb{D C} J_{1}}{2} \text {. }
$$

Proof. By Theorem 12 for $r=0$ we have

$$
\begin{aligned}
& \sum_{l=0}^{n} \mathbb{D} \mathbb{C} J(0, l)= \frac{\mathbb{D} \mathbb{J}(0, n+1)+2 \mathbb{D} \mathbb{C}(0, n)-3(1+i+\varepsilon+i \varepsilon)}{2} \\
&= \frac{-(i+4 \varepsilon+9 i \varepsilon)}{\mathbb{D} J(0, n+2)-(3+5 i+11 \varepsilon+21 i \varepsilon)} \\
& 2
\end{aligned}
$$

Using fact that $J(0, n)=J_{n+2}$ and $\mathbb{D} \mathbb{C} J_{0}=i+\varepsilon+3 i \varepsilon, \mathbb{D} J_{1}=1+i+3 \varepsilon+5 i \varepsilon$, we get

$$
\begin{aligned}
\sum_{l=0}^{n} \mathbb{D} \mathbb{C} J_{l} & =\frac{\mathbb{D} J_{n+2}-(3+5 i+11 \varepsilon+21 i \varepsilon)}{2}+\mathbb{D} \mathbb{C} J_{0}+\mathbb{D} \mathbb{C} J_{1} \\
& =\frac{\mathbb{D} J_{n+2}-(3+5 i+11 \varepsilon+21 i \varepsilon)+2(1+2 i+4 \varepsilon+8 i \varepsilon)}{2} . \\
& =\frac{\mathbb{D} J_{n+2}-(1+i+3 \varepsilon+5 i \varepsilon)}{2}=\frac{\mathbb{D} J_{n+2}-\mathbb{D} \mathbb{C} J_{1}}{2},
\end{aligned}
$$


which ends the proof.

The next theorem gives the Honsberger type identity for the dual-complex r-Jacobsthal numbers.

Theorem 13 Let $m \geq 2, n \geq 1, r \geq 0$ be integers. Then

$$
\begin{aligned}
& 2^{r} \mathbb{D} \mathbb{C J}(r, m-1) \cdot \mathbb{D} \mathbb{C J}(r, n)+\left(4^{r}+8^{r}\right) \mathbb{D} \mathbb{C J}(r, m-2) \cdot \mathbb{D C J}(r, n-1)= \\
& =2 \mathbb{C} J(r, m+n)-J(r, m+n)-J(r, m+n+2) \\
& \quad-2 \varepsilon J(r, m+n+4)+2 i \varepsilon J(r, m+n+3) .
\end{aligned}
$$

Proof. By simple calculations we have

$$
\begin{aligned}
& 2^{r} \mathbb{D} \mathbb{C} J(r, m-1) \cdot \mathbb{D} \mathbb{C} J(r, n)= \\
& =2^{r}[J(r, m-1) J(r, n)+i J(r, m-1) J(r, n+1) \\
& \quad+\varepsilon J(r, m-1) J(r, n+2)+i \varepsilon J(r, m-1) J(r, n+3) \\
& \quad+i J(r, m) J(r, n)-J(r, m) J(r, n+1)+i \varepsilon J(r, m) J(r, n+2) \\
& \quad-\varepsilon J(r, m) J(r, n+3)+\varepsilon J(r, m+1) J(r, n)+i \varepsilon J(r, m+1) J(r, n+1) \\
& \quad+i \varepsilon J(r, m+2) J(r, n)-\varepsilon J(r, m+2) J(r, n+1)] \\
& \left(4^{r}+8^{r}\right) \mathbb{D} \mathbb{C} J(r, m-2) \cdot \mathbb{D} \mathbb{C} J(r, n-1)= \\
& =\left(4^{r}+8^{r}\right)[J(r, m-2) J(r, n-1)+i J(r, m-2) J(r, n) \\
& \quad+\varepsilon J(r, m-2) J(r, n+1)+i \varepsilon J(r, m-2) J(r, n+2) \\
& \quad+i J(r, m-1) J(r, n-1)-J(r, m-1) J(r, n) \\
& \quad+i \varepsilon J(r, m-1) J(r, n+1)-\varepsilon J(r, m-1) J(r, n+2) \\
& \quad+\varepsilon J(r, m) J(r, n-1)+i \varepsilon J(r, m) J(r, n) \\
& \quad+i \varepsilon J(r, m+1) J(r, n-1)-\varepsilon J(r, m+1) J(r, n)] .
\end{aligned}
$$

Hence

$$
\begin{aligned}
& 2^{r} \cdot \mathbb{D} \mathbb{C} J(r, m-1) \cdot \mathbb{D} \mathbb{C} J(r, n)+\left(4^{r}+8^{r}\right) \mathbb{D} \mathbb{C} J(r, m-2) \cdot \mathbb{D} \mathbb{C} J(r, n-1)= \\
& =2^{r} J(r, m-1) J(r, n)+\left(4^{r}+8^{r}\right) J(r, m-2) J(r, n-1) \\
& \quad+i\left[2^{r} J(r, m-1) J(r, n+1)+\left(4^{r}+8^{r}\right) J(r, m-2) J(r, n)\right. \\
& \left.\quad+2^{r} J(r, m) J(r, n)+\left(4^{r}+8^{r}\right) J(r, m-1) J(r, n-1)\right] \\
& \quad+\varepsilon\left[2^{r} J(r, m-1) J(r, n+2)+\left(4^{r}+8^{r}\right) J(r, m-2) J(r, n+1)\right. \\
& \left.\quad+2^{r} J(r, m+1) J(r, n)+\left(4^{r}+8^{r}\right) J(r, m) J(r, n-1)\right] \\
& \quad+i \varepsilon\left[2^{r} J(r, m-1) J(r, n+3)+\left(4^{r}+8^{r}\right) J(r, m-2) J(r, n+2)\right. \\
& \left.\quad+2^{r} J(r, m) J(r, n+2)+\left(4^{r}+8^{r}\right) J(r, m-1) J(r, n+1)\right] \\
& \quad-2^{r} J(r, m) J(r, n+1)-\left(4^{r}+8^{r}\right) J(r, m-1) J(r, n) \\
& \quad-\varepsilon\left[2^{r} J(r, m) J(r, n+3)+\left(4^{r}+8^{r}\right) J(r, m-1) J(r, n+2)\right. \\
& \left.+2^{r} J(r, m+2) J(r, n+1)+\left(4^{r}+8^{r}\right) J(r, m+1) J(r, n)\right] \\
& \quad+i \varepsilon\left[2^{r} J(r, m+1) J(r, n+1)+\left(4^{r}+8^{r}\right) J(r, m) J(r, n)\right. \\
& \left.\quad+2^{r} J(r, m+2) J(r, n)+\left(4^{r}+8^{r}\right) J(r, m+1) J(r, n-1)\right] .
\end{aligned}
$$


Using Theorem 4, we get

$$
\begin{aligned}
& 2^{r} \mathbb{D} \mathbb{C J}(r, m-1) \cdot \mathbb{D} \mathbb{C} J(r, n)+\left(4^{r}+8^{r}\right) \mathbb{D} \mathbb{C} J(r, m-2) \cdot \mathbb{D} \mathbb{C} J(r, n-1)= \\
& =J(r, m+n)+2[i J(r, m+n+1)+\varepsilon J(r, m+n+2) \\
& \quad+i \varepsilon J(r, m+n+3)]-J(r, m+n+2) \\
& \quad-2 \varepsilon J(r, m+n+4) \varepsilon+2 i \varepsilon J(r, m+n+3) \\
& =2 \mathbb{D} \mathbb{C} J(r, m+n)-J(r, m+n)-J(r, m+n+2) \\
& \quad-2 \varepsilon J(r, m+n+4)+2 i \varepsilon J(r, m+n+3) .
\end{aligned}
$$

\section{$5 \quad$ Generating functions and matrix generators}

Now we give the generating function of the dual-complex r-Jacobsthal numbers.

Theorem 14 The generating function of the dual-complex $r$-Jacobsthal numbers has the following form

$$
g(x)=\frac{\mathbb{D} \mathbb{C} J(r, 0)+\left(\mathbb{D} \mathbb{C} J(r, 1)-2^{r} \mathbb{D} \mathbb{C} J(r, 0)\right) x}{1-2^{r} x-\left(2^{r}+4^{r}\right) x^{2}} .
$$

Proof. Let

$$
g(x)=\mathbb{D} \mathbb{C} J(r, 0)+\mathbb{D} \mathbb{C} J(r, 1) x+\mathbb{D} \mathbb{C} J(r, 2) x^{2}+\cdots+\mathbb{D} \mathbb{C}(r, n) x^{n}+\cdots
$$

be the generating function of the dual-complex $r$-Jacobsthal numbers. Then

$$
\begin{aligned}
2^{r} x g(x)= & 2^{r} \mathbb{D} \mathbb{C} J(r, 0) x+2^{r} \mathbb{D} \mathbb{C J}(r, 1) x^{2}+2^{r} \mathbb{D} \mathbb{C J}(r, 2) x^{3} \\
& +\cdots+2^{r} \mathbb{D} \mathbb{C J}(r, n-1) x^{n}+\cdots \\
\left(2^{r}+4^{r}\right) x^{2} g(x)= & \left(2^{r}+4^{r}\right) \mathbb{D} \mathbb{C} J(r, 0) x^{2}+\left(2^{r}+4^{r}\right) \mathbb{D} \mathbb{C J}(r, 1) x^{3} \\
& +\left(2^{r}+4^{r}\right) \mathbb{D} \mathbb{C} J(r, 2) x^{4}+\cdots \\
& +\left(2^{r}+4^{r}\right) \mathbb{D} \mathbb{C J}(r, n-2) x^{n}+\cdots
\end{aligned}
$$

By Proposition 2 we get

$$
\begin{aligned}
& g(x)-2^{r} x g(x)-\left(2^{r}+4^{r}\right) x^{2} g(x) \\
& =\mathbb{D} \mathbb{C}(r, 0)+\left(\mathbb{D} \mathbb{C}(r, 1)-2^{r} \mathbb{D} \mathbb{C} J(r, 0)\right) x \\
& \quad+\left(\mathbb{D} \mathbb{C} J(r, 2)-2^{r} \mathbb{D} \mathbb{C}(r, 1)-\left(2^{r}+4^{r}\right) \mathbb{D} \mathbb{C} J(r, 0)\right) x^{2}+\cdots \\
& =\mathbb{D} \mathbb{C J}(r, 0)+\left(\mathbb{D} \mathbb{C} J(r, 1)-2^{r} \mathbb{D} \mathbb{C} J(r, 0)\right) x .
\end{aligned}
$$


Thus

$$
g(x)=\frac{\mathbb{D} \mathbb{C} J(r, 0)+\left(\mathbb{D} \mathbb{C} J(r, 1)-2^{r} \mathbb{D} \mathbb{C} J(r, 0)\right) x}{1-2^{r} x-\left(2^{r}+4^{r}\right) x^{2}} .
$$

Moreover,

$$
\begin{aligned}
\mathbb{D C J}(r, 0)= & 1+\left(2^{r+1}+1\right) i+\left(3 \cdot 4^{r}+2^{r+1}\right) \varepsilon \\
& +\left(5 \cdot 8^{r}+5 \cdot 4^{r}+2^{r}\right) i \varepsilon \\
\mathbb{D C J}(r, 1)-2^{r} \mathbb{D C} J(r, 0)= & 2^{r}+1+\left(4^{r}+2^{r}\right) i+\left(2 \cdot 8^{r}+3 \cdot 4^{r}+2^{r}\right) \varepsilon \\
& +\left(3 \cdot 16^{r}+5 \cdot 8^{r}+2 \cdot 4^{r}\right) i \varepsilon .
\end{aligned}
$$

Corollary 7 The generating function of the dual-complex Jacobsthal sequence is

$$
g(x)=\frac{i+\varepsilon+3 i \varepsilon+(1+2 \varepsilon+2 i \varepsilon) x}{1-x-2 x^{2}} .
$$

At the end we give the matrix representation of the dual-complex r-Jacobsthal numbers.

Theorem 15 Let $\mathrm{n} \geq 1, \mathrm{r} \geq 0$ be integers. Then

$$
\left[\begin{array}{ll}
\mathbb{D} C J(r, n+1) & \mathbb{D} \mathbb{C J}(r, n) \\
\mathbb{D} \mathbb{C} J(r, n) & \mathbb{D} \mathbb{C J}(r, n-1)
\end{array}\right]=\left[\begin{array}{ll}
\mathbb{D} \mathbb{C} J(r, 2) & \mathbb{D} \mathbb{C J}(r, 1) \\
\mathbb{D} \mathbb{C} J(r, 1) & \mathbb{D} \mathbb{C J}(r, 0)
\end{array}\right] \cdot\left[\begin{array}{ll}
2^{r} & 1 \\
2^{r}+4^{r} & 0
\end{array}\right]^{n-1} .
$$

Proof. (by induction on $n$ ) It is easy to check that for $n=1$ the result holds. Assume that the formula (7) is true for $n \geq 1$. We will show that

$$
\left[\begin{array}{ll}
\mathbb{D C} J(r, n+2) & \mathbb{D} \mathbb{C J}(r, n+1) \\
\mathbb{D} \mathbb{C} J(r, n+1) & \mathbb{D} \mathbb{C J}(r, n)
\end{array}\right]=\left[\begin{array}{ll}
\mathbb{D} \mathbb{C} J(r, 2) & \mathbb{D} \mathbb{C} J(r, 1) \\
\mathbb{D} \mathbb{C J}(r, 1) & \mathbb{D} \mathbb{C J}(r, 0)
\end{array}\right] \cdot\left[\begin{array}{ll}
2^{r} & 1 \\
2^{r}+4^{r} & 0
\end{array}\right]^{n} .
$$

By induction's hypothesis and simple calculations we have

$$
\begin{aligned}
& {\left[\begin{array}{ll}
\mathbb{D} \mathbb{C J}(r, 2) & \mathbb{D} \mathbb{C J}(r, 1) \\
\mathbb{D C J}(r, 1) & \mathbb{D} \mathbb{C J}(r, 0)
\end{array}\right] \cdot\left[\begin{array}{ll}
2^{r} & 1 \\
2^{r}+4^{r} & 0
\end{array}\right]^{n-1} \cdot\left[\begin{array}{ll}
2^{r} & 1 \\
2^{r}+4^{r} & 0
\end{array}\right]} \\
& =\left[\begin{array}{ll}
\mathbb{D} \mathbb{C J}(r, n+1) & \mathbb{D} \mathbb{C J}(r, n) \\
\mathbb{D C J}(r, n) & \mathbb{D} \mathbb{C J}(r, n-1)
\end{array}\right] \cdot\left[\begin{array}{ll}
2^{r} & 1 \\
2^{r}+4^{r} & 0
\end{array}\right] \\
& =\left[\begin{array}{ll}
2^{r} \mathbb{D} \mathbb{C J}(r, n+1)+\left(2^{r}+4^{r}\right) \mathbb{D} \mathbb{C} J(r, n) & \mathbb{D} \mathbb{C} J(r, n+1) \\
2^{r} \mathbb{D} \mathbb{C J}(r, n)+\left(2^{r}+4^{r}\right) \mathbb{D} \mathbb{C J}(r, n-1) & \mathbb{D} \mathbb{C J}(r, n)
\end{array}\right]
\end{aligned}
$$




$$
=\left[\begin{array}{ll}
\mathbb{D} \mathbb{C J}(r, n+2) & \mathbb{D} \mathbb{C} J(r, n+1) \\
\mathbb{D} \mathbb{C J}(r, n+1) & \mathbb{D} \mathbb{C J}(r, n)
\end{array}\right],
$$

which ends the proof.

Calculating the determinants in formula (7) we obtain the Cassini type identity for the dual-complex r-Jacobsthal numbers. We have

$$
\begin{aligned}
& \begin{array}{ll}
\mathbb{D C J}(r, n+1) & \mathbb{D C J}(r, n) \\
\mathbb{D C J}(r, n) & \mathbb{D} \mathbb{C J}(r, n-1)
\end{array} \mid=\mathbb{D} \mathbb{C J}(r, n+1) \cdot \mathbb{D} \mathbb{C J}(r, n-1)-(\mathbb{D} \mathbb{C J}(r, n))^{2}, \\
& \begin{array}{ll}
\mathbb{D} \mathbb{C J}(r, 2) & \mathbb{D} \mathbb{C J}(r, 1) \\
\mathbb{D} \mathbb{C J}(r, 1) & \mathbb{D} \mathbb{C J}(r, 0)
\end{array} \mid=\mathbb{D} \mathbb{C J}(r, 2) \cdot \mathbb{D} \mathbb{C J}(r, 0)-(\mathbb{D} \mathbb{C J}(r, 1))^{2} . \\
& \left|\begin{array}{ll}
2^{r} & 1 \\
2^{r}+4^{r} & 0
\end{array}\right|^{n-1}=\left(-\left(2^{r}+4^{r}\right)\right)^{n-1} .
\end{aligned}
$$

Consequently,

$$
\begin{aligned}
& \mathbb{D} \mathbb{C J}(r, n+1) \cdot \mathbb{D} \mathbb{C J}(r, n-1)-(\mathbb{D} \mathbb{C J}(r, n))^{2}= \\
& =\left(-\left(2^{r}+4^{r}\right)\right)^{n-1}\left(\mathbb{D} \mathbb{C J}(r, 2) \cdot \mathbb{D} \mathbb{C J}(r, 0)-(\mathbb{D} \mathbb{C} J(r, 1))^{2}\right) .
\end{aligned}
$$

\section{Compliance with ethical standards}

Conflict of Interest: The authors declare that they have no conflict of interest.

\section{References}

[1] M. Akar, S. Yüce, S. Şahin, On the Dual Hyperbolic Numbers and the Complex Hyperbolic Numbers, Journal of Computer Science \& Computational Mathematics, 8(1) (2018) DOI: 10.20967/jcscm.2018.01.001.

[2] F. T. Aydin, Dual-complex k-Fibonacci numbers, Chaos Solitons Fractals, 115 (2018), 1-6.

[3] F. T. Aydin, Dual-complex k-Pell quaternions, Notes on Number Theory and Discrete Mathematics, vol. 25, no. 3 (2019), 111-125.

[4] D. Bród, On a new Jacobsthal-type sequence, Ars Combin., 150 (2020), 21-29.

[5] W. K. Clifford, Preliminary Sketch of Biquaternions, Proc. Lond. Math. Soc., s1-4 (1873), 381-395. 
[6] M. A. Güungör, A. Z. Azak, Investigation of Dual-Complex Fibonacci, Dual-Complex Lucas Numbers and Their Properties, Adv. Appl. Clifford Algebr., 27 (2017), 3083-3096.

[7] A. F. Horadam, Complex Fibonacci Numbers and Fibonacci Quaternions, Amer. Math. Monthly, 70 (1963), 289-291.

[8] V. Majernik, Multicomponent number systems, Acta Phys. Polon. A, 90 (3) (1996), 491-498.

[9] A. Szynal-Liana, I. Włoch, A note on Jacobsthal quaternions, Adv. Appl. Clifford Algebr., 26 (2016), 441-447.

[10] A. Szynal-Liana, I. Włoch, The Pell quaternions and the Pell octonions, Adv. Appl. Clifford Algebr., 26 (2016), 435-440.

Received: December 21, 2020 